\title{
LETTER OPEN \\ L1 drives HSC aging and affects prognosis of chronic myelomonocytic leukemia
}

\author{
Signal Transduction and Targeted Therapy (2020)5:205
}

\section{Dear Editor,}

Telomere attrition is one of the hallmark of aging. Lategeneration Terc knockout mice exhibit impaired hematopoiesis, ${ }^{1}$ while the underling mechanisms remain poorly understood. Retrotransposon long interspersed element-1 (L1) is the only human retrotransposable elements capable of autonomous retrotransposition, and evolutionarily inactive. Recent studies reported that $L 1$ is derepressed during the aging process with redistribution and reorganization of the heterochromatin. ${ }^{2}$ Considering that telomere shortening can cause chromosome instability and rearrangements, ${ }^{3}$ we speculate that $L 1$ may play a role in impaired hematopoiesis in telomere dysfunctional mice.

As expected, we found that the expression of $L 1$ were significantly increased in bone marrow (BM) cells from the 3rd generation telomerase deficient $\left(\mathrm{G} \mathrm{Terc}^{-/-}\right)$mice compared with that of wild type (WT) mice (Fig. 1a, Supplementary Fig. S1a), which was associated with the decreased CpG methylation at L1 promoter region in $\mathrm{G} \mathrm{Terc}^{-/-}$mice (Supplementary Fig. S1b). Additionally, we observed that $\mathrm{L} 1$ was accumulated in cytosol of BM cells in G3Terc ${ }^{-1-}$ mice (Fig. 1b, Supplementary Fig. S2a). To determine whether L1 activates cGAS signaling in mice with telomere dysfunction, we examined 2' 3 '-cGAMP expression and the phosphorylation levels of TBK1, IRF3, and NF-KB p65, the canonical 2'3'-cGAMP downstream, and found that CGAS signaling was significantly activated(Fig. 1c, Supplementary Fig. S2b). Consequently, the expression of type I interferon IFNa and IFN $\beta$, downstream interferon-stimulated genes (CXCL1 and CXCL10), and other cytokines (IL-6, IL-17A, and TNFa) were dramatically upregulated in BM cells and plasma in $\mathrm{G} \mathrm{Terc}^{-/-}$mice (Fig. 1d, Supplementary Fig. S2c, d). Furthermore, we generated G3Terc ${ }^{-1}$ ${ }_{-} \mathrm{GAS}^{-1-}$ double knockout mice, and found the reduced type I interferon response and decreased expression of cytokines in $\mathrm{G} \mathrm{Terc}^{-1-} \mathrm{CGAS}^{-1-}$ mice (Fig. 1e, Supplementary Fig. S2e, f). These results demonstrate that L1-CGAS signaling is responsible for telomere dysfunction induced inflammation.

To further illustrate the critical function of $L 1$ in regulating cGAS signaling and HSC function, we administered the $\mathrm{L} 1$ reverse transcription inhibitor 3TC to G3Terc ${ }^{-1-}$ mice (Supplementary Fig. S3a). We found that the cytosolic accumulation of L1 CDNA (Fig. If, Supplementary Fig. S3b), the expression of 2'3'-cGAMP (Fig. 1g), and the phosphorylation levels of TBK1, IRF3, and NF-KB p65 were significantly decreased in $\mathrm{G} \mathrm{Terc}^{-/-}$mice treated with 3TC (Supplementary Fig. S3c), but the cytosolic accumulation of L1 CDNA and the CGAS signaling was not affected in WT mice treated with 3TC (Fig. 1f-g, Supplementary Fig. S3b, d). Consequently, the cytokines production were dramatically reduced in BM cells and plasma of G3Terc ${ }^{-1-}$ mice treated with 3TC, but not in that of WT mice treated with 3TC(Fig. 1h, Supplementary Fig. S3e, f). Furthermore, Depletion of L1 significantly decreased cytokines expression in BM cells of $\mathrm{G}^{2} \mathrm{Terc}^{-1-}$ mice (Fig. 1i). To verify whether reduced inflammation by suppression of $L 1$ could rescue

\author{
; https://doi.org/10.1038/s41392-020-00279-4
}

the impaired HSC maintenance and function in telomere dysfunctional mice as we previous reported, ${ }^{1}$ we performed flow cytometry analysis and the results showed that 3TC treatment could recover the frequency of long-term HSCs (LTs) and multipotential progenitors (MPPs) in telomere dysfunctional mice, and 3TC treatment had no effect on the proportion of HSCs in WT mice (Fig. 1j, Supplementary Fig. S4a). Notably, competitive transplantation experiment revealed a significant improvement in the repopulating capacity of HSCs isolated from $\mathrm{G}_{3} \mathrm{Terc}^{-1-}$ mice treated with 3TC, but without affected the lineage distribution at steady state (Fig. 1k-l, Supplementary Fig. S4b). Together, these results demonstrate that suppression of $\mathrm{L} 1$ alleviates inflammation, thereby improves the HSC maintenance and function.

In human, chronic myelomonocytic leukemia (CMML) is a chronic myeloid neoplasm of the elderly with a poor prognosis. Mice harboring an oncogenic G12D mutation in Nras locus (Nras ${ }^{G 12 D}$ mice) can develop a CMML-like disease. Recent study reported that inflammatory cytokines are elevated in CMML patients. ${ }^{4}$ To determine whether the telomere dysfunction induced inflammatory environment affects the prognosis of leukemia, we established a mouse BM transplantation model by using the BM from Nras ${ }^{\text {G12D }}$ mice as donor, and half lethal dose irradiated G3Terc $^{-1-}$ or WT mice as recipients (Supplementary Fig. S5a). We found that both G3Terc ${ }^{-1-}$ and WT mice transplanted with Nras ${ }^{G 12 D}$ BM (G3Terc ${ }^{-1-}$ recipient mice and WT recipient mice) could develop a CMML-like disease, and observed that diseased mice showed enhanced hepatosplenomegaly compared to control mice (Supplementary Fig. S5b, c), and gradually increased frequency of myeloid cells in peripheral blood (PB) from both WT recipient mice and $\mathrm{G} \operatorname{Terc}^{-1-}$ recipient mice (Supplementary Fig. S5d). Notably, the G3Terc ${ }^{-1-}$ recipient mice showed significantly reduced survival compared with WT recipient mice (Fig. $1 \mathrm{~m}$ ). The white blood cells count and the frequency of neutrophils were significantly increased, whereas the red blood cells count, platelets count, and frequency of lymphocytes were decreased in $\mathrm{G}^{2} \operatorname{Terc}^{-1-}$ recipient mice (Supplementary Fig. S5e). The BM analysis showed that absolute number of $T$ and $B$ lymphocytes were significantly decreased in $\mathrm{G} \mathrm{Terc}^{-1-}$ recipient mice (Fig. 1n). The absolute number and frequency of recipientderived LTs, megakaryocytic/ erythroid progenitors (MEPs) and common lymphoid progenitors (CLPs) were decreased in $\mathrm{G} \mathrm{TerC}^{-1-}$ recipient mice, and the absolute number and frequency of donor-derived common myeloid progenitors (CMPs), MEPs and CLPs were decreased in G3Terc ${ }^{-1-}$ recipient mice as well (Fig. 10, $\mathrm{p}$, Supplementary Fig. S5f-h). In addition, we found that the cytokines production were dramatically upregulated in plasma of $\mathrm{G}$ Terc $^{-1-}$ recipient mice, although IL-6 was also increased in donor mice (Supplementary Fig. S5i). Together, these results indicate that the repression of the normal hematopoiesis and increased inflammation contributes to the decreased survival of

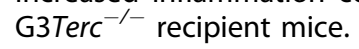


a

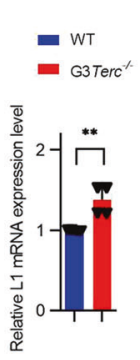

f

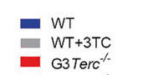

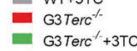

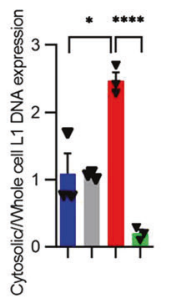

k

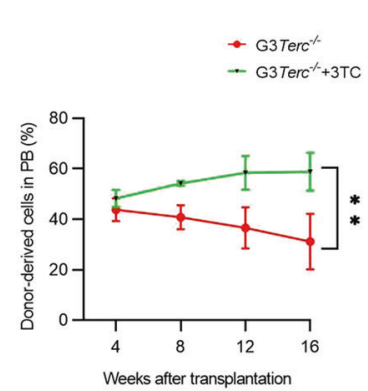

o

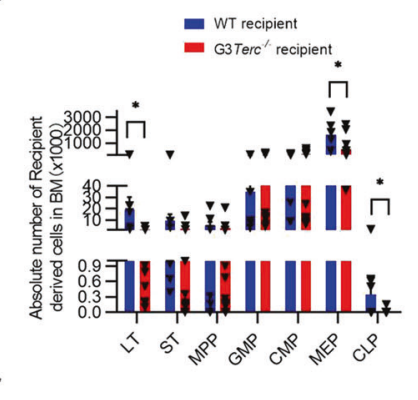

- G3Terc" recipient

- G3Terc ${ }^{-1}$ recipient +3TC

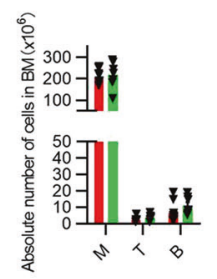

b

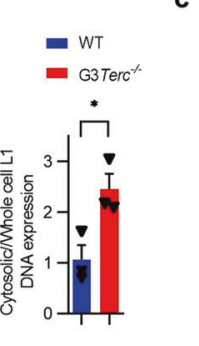

c

$$
\text { = }{ }^{W T}{ }^{\text {G }} \text { Terc }^{\circ}
$$

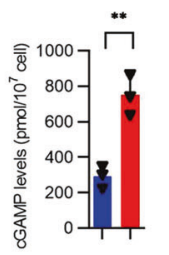

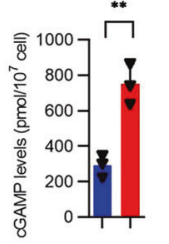

d

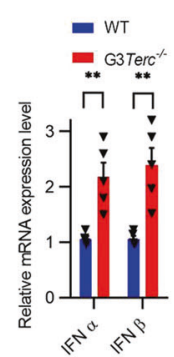

e

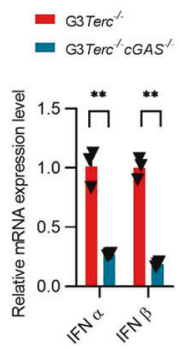

h

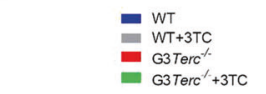

i

j

G3Terc's +siControl

G3Terc's +siL1

르 ${ }^{W T+3 T C}$

E3 G Tere $^{-1}$
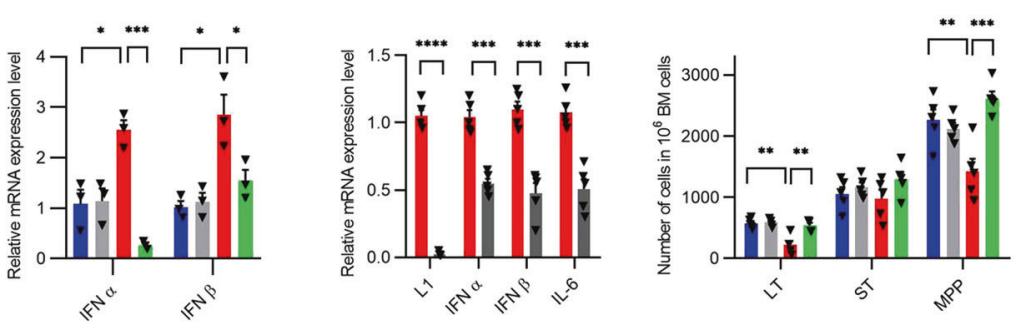

I $\mathbf{m}$

$$
\text { G3Terc'sTC }
$$

- WT recipient

n

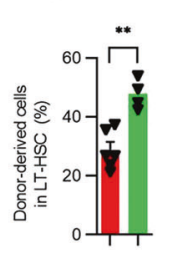

- Gs Terc recipient

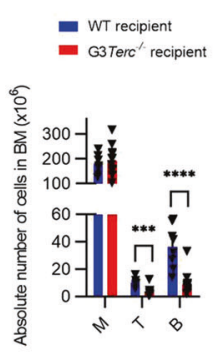

p

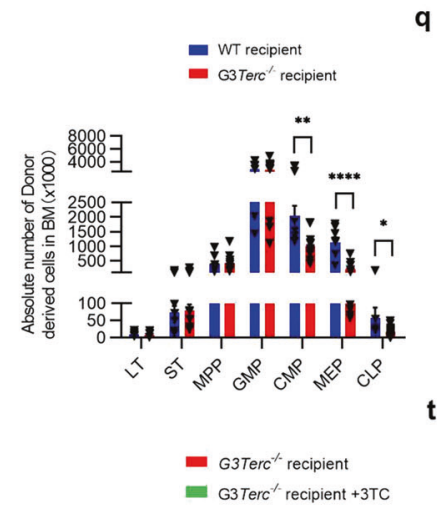

$\mathbf{q}$

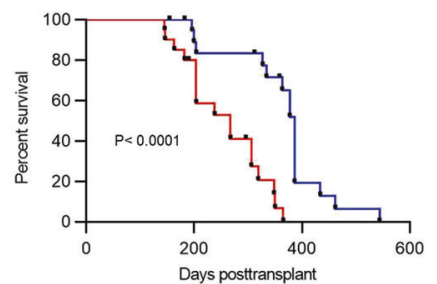

q

- WT recipient

- WT recipient $+3 T C$ + G3Terc recipient

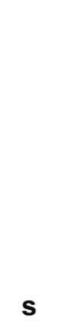

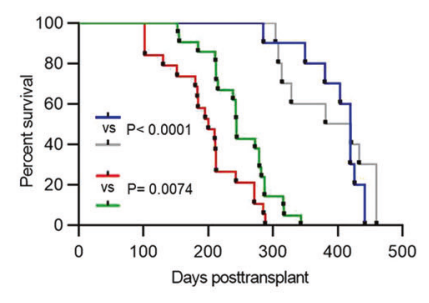

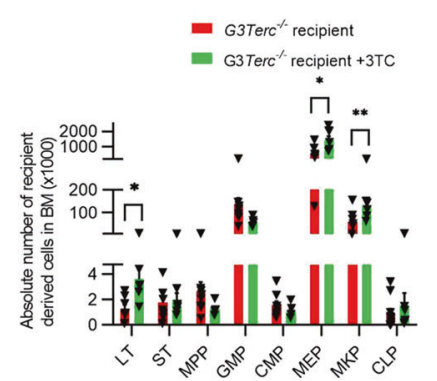

To determine whether improvement of HSC by L1 inhibition is beneficial in prognosis of CMML, we administered 3TC to $\mathrm{G} \mathrm{Terc}^{-1-}$ recipient mice and WT recipient mice (Supplementary Fig. S6a), and found that the 3TC treatment extended the survival of G3Terc ${ }^{-1-}$ recipient mice, but not WT recipient mice (Fig. 1q).
The expression of cytokines in plasma of ${\mathrm{G} 3 \mathrm{Terc}^{-/-}}^{-}$recipient mice treated with 3TC were significantly decreased (Supplementary Fig. S6b). Interestingly, we found that 3TC could not relieve the hepatosplenomegaly, high proportion of myeloid cells in PB and high absolute number of myeloid cells in BM from G3Terc $^{-1-}$ 
Fig. 1 L1 drives HSC aging and affects prognosis of chronic myelomonocytic leukemia. a Quantitative real-time PCR (Q-PCR) analysis of the relative mRNA expression level of $\mathrm{L} 1$ in BM of WT and G3Terc ${ }^{-1}$ mice $(n=4$, left). The protein levels of $\mathrm{L} 1$ (ORF1p) was determined by western blot in BM of WT and G3Terc ${ }^{-1-}$ mice. The graph represents the relative ORF1p protein abundance (right). b Q-PCR analysis of the relative CDNA levels of L1 in BM cytoplasmic fraction of WT and G3Terc ${ }^{-1-}$ mice $(n=3)$. c $2^{\prime} 3^{\prime}-$ cGAMP levels were determined by LC-MS/MS in BM of WT and G3Terc ${ }^{-1-}$ mice $(n=3)$. d Q-PCR analysis of the relative mRNA expression levels of IFN $\alpha$ and IFN $\beta$ in WT and G3Terc ${ }^{-1-}$ mice $(n=5)$. e Q-PCR analysis of the relative mRNA expression levels of IFN $\alpha$ and IFN $\beta$ in G3Terc ${ }^{-1-}$ and G3Terc ${ }^{-1-} \mathrm{cGAS}^{-1-}$ mice $(n=3)$. $\mathbf{f} \mathrm{Q}-\mathrm{PCR}$ analysis of the relative cDNA levels of L1 in BM cytoplasmic fraction of WT or G3Terc ${ }^{-1}$ mice treated with or without 3TC $(n=3)$. g 2'3'-cGAMP levels were determined by LC-MS/MS in BM of WT or G3Terc ${ }^{-1-}$ mice treated with or without 3TC $(n \geq 5)$. $\mathbf{h}$ Q-PCR analysis of the relative mRNA expression levels of IFN $\alpha$ and IFN $\beta$ in BM of WT or G3Terc ${ }^{-1-}$ mice treated with or without $3 T C(n=3)$. i Q-PCR analysis of relative mRNA expression levels of L1, IFN $\alpha$, IFN $\beta$, and IL- 6 in control and L1 depleted BM cells of G3Terc ${ }^{-1-}$ mice $(n=5)$. j Numbers of LT (CD34 ${ }^{-}$Flt $3^{-}$LSK), ST $\left(\mathrm{CD} 34^{+} \mathrm{Flt} 3^{-}\right.$LSK) and MPP (CD34 ${ }^{+} \mathrm{FIt}^{+}$LSK) per million BM cells of WT, or G3Terc ${ }^{-1-}$ mice treated with or without 3TC $(n=5)$. $\mathbf{k}$ Percentage of donor-derived PB cells of G3Terc ${ }^{-1-}$ mice treated with or without 3TC at the indicated time points in competitive transplantation assay ( $n \geq 3$ ). I Percentage of donor-derived LT in BM of G3Terc ${ }^{-/-}$mice and 3TC-treated G3Terc ${ }^{-/-}$mice 16 weeks after transplantation ( $\left.n \geq 4\right)$. $\mathbf{m}$ Survival curve of WT recipient mice and G3Terc ${ }^{-1-}$ recipient mice following transplantation $(n=20)$. $\mathbf{n}$ Absolute number of M, T, and B cells in BM of WT recipient mice and G3Terc ${ }^{-1}$ recipient mice $(n \geq 8)$. o Absolute number of LT, ST, MPP, GMP (granulocyte/monocyte progenitor

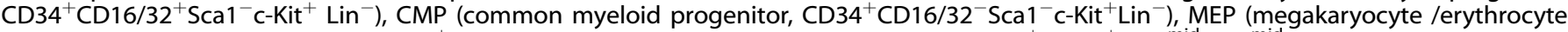

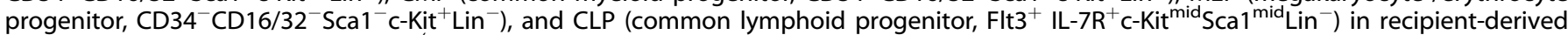
BM of WT recipient mice and G3Terc ${ }^{-1-}$ recipient mice $(n \geq 7)$. p Absolute number of LT, ST, MPP, GMP, CMP, MEP, and CLP in donor-derived BM of WT recipient mice and G3Terc ${ }^{-1-}$ recipient mice $(n \geq 5)$. q Survival curve of WT recipient or G3Terc ${ }^{-1-}$ recipient mice treated with or without 3TC following transplantation $(n \geq 10)$. r Absolute number of M, T, and B cells in BM of G3Terc ${ }^{-1-}$ recipient mice treated with or without 3TC $(n \geq 7)$. s Absolute number of LT, ST, MPP, GMP, CMP, MEP, MKP (IL-7R ${ }^{-} \mathrm{CD} 41^{+} \mathrm{C}_{-} \mathrm{Kit}^{+} \mathrm{Lin}^{-}$) and CLP in donor-derived BM of G3Terc ${ }^{-/-}$recipient mice treated with or without 3TC $(n \geq 6)$. t Absolute number of LT, ST, MPP, GMP, CMP, MEP, MKP, and CLP in recipient-derived BM of G3Terc ${ }^{-/-}$ recipient mice treated with or without $3 \mathrm{TC}(n \geq 7) .{ }^{*} p<0.05 ;{ }^{* *} p<0.01 ;{ }^{* * *} p<0.001 ;{ }^{* * *} p<0.0001$

recipient mice (Fig. 1r, Supplementary Fig. S6c-e), and the absolute number and frequency of donor-derived hematopoietic stem/ progenitor cells (HSPCs) were comparable between 3TC treated and untreated $\mathrm{G} \mathrm{Terc}^{-1-}$ recipient mice (Fig. 1s, Supplementary Fig. S6f). Notably, the absolute number and frequency of recipientderived LTs, MEPs and megakaryocyte progenitors (MKPs) were dramatically increased, and the PLTs count was rescued to normal level in $\mathrm{G}$ Terc $^{-1-}$ recipient mice with $3 \mathrm{TC}$ treatment (Fig. 1t, Supplementary Fig. S6g, h). To explore whether 3TC treatment also contributes to the repression of donor cell (Nras ${ }^{G 12 D}$ ), we checked the mRNA expression and CpG methylation level of L1 in diseased mice. The results showed decreased $\mathrm{CpG}$ methylation at L1 promoter region in endogenous $\mathrm{G} \mathrm{Terc}^{-1-} \mathrm{BM}$ cells, but not in endogenous WT BM cells, the donor-derived (Nras ${ }^{G 12 D}$ ) BM cells in $\mathrm{WT}$ and $\mathrm{G} \mathrm{Terc}^{-1-}$ recipient mice (Supplementary Fig. S7a). The expression level of $\mathrm{L} 1$ in different groups were consistent with the CpG methylation levels (Supplementary Fig. S7b). Altogether, these results indicate that the suppression of L1 by 3TC treatment in CMML mice reduces telomere dysfunction induced inflammation and attenuates the impaired hematopoiesis, thereby extended survival of the diseased mice, probably due to the improvement of HSPCs maintenance.

In summary, we found that L1 activation is responsible for the cGAS signaling induced inflammatory responses in G3Terc $^{-/-}$ mice. 3TC treatment attenuated the aging-associated decline of HSC maintenance and function, thereby extended the survival of $\mathrm{G} \mathrm{Terc}^{-/-}$recipient mice transplanted with oncogenic Nras ${ }^{G 12 D}$ BM. Our findings suggest that reverse transcriptase inhibition may serve as a new therapeutic strategy for patients suffering from age-related disorders.

\section{ACKNOWLEDGEMENTS}

We thank the Dr. Jian Mao (School of Medicine, Hangzhou Normal University) for technical assistance. This work was supported by Grants 2016YFA0100602, 2017YFA0103302, 2018YFA0109300 from the National Key Research and Development Program of China; Grants 81525010, 91749203, 81871116, 81501214, 91749117, 81770155 , and 81771502 from the National Natural Science Foundation of China; Grants LQ14C070002 from the Natural Science Foundation of Zhejiang Province of China; Grant 2018GZR110103002 from Innovative Team Program of Guangzhou Regenerative Medicine and Health Guangdong Laboratory and Grant 2017ZT07S347 from the Program for Guangdong Introducing Innovative and Enterpreneurial Teams. This work was supported by the Science Foundation for Distinguished Young Scholars of Guangdong Province (2019B151502008) to Hu Wang.

\section{AUTHOR CONTRIBUTIONS}

Y.W., JP.Z., H.W., Y.L., JY.W., and LJ.X. carried out the experiments. Y.W., JP.Z., H.W., JinY W., J.M.S., ZF.S., and ZY.J. analyzed the data. Y.W., JP.Z., H.W., J.M.S., and ZY.J. wrote the paper.

\section{ADDITIONAL INFORMATION}

The online version of this article (https://doi.org/10.1038/s41392-020-00279-4) contains supplementary material, which is available to authorized users.

Competing interests: The authors declare no competing interests.

Ying Wang ${ }^{1}$, Jin-ping Zheng ${ }^{2}$, Ying Luo ${ }^{3}$, Junyi Wang ${ }^{1}$, Lingjie $\mathrm{Xu}^{1}$, Jinyong Wang ${ }^{4}$, John M. Sedivy ${ }^{5}$, Zhangfa Song ${ }^{6}$, Hu Wang ${ }^{1,3}$ and Zhenyu Ju',

${ }^{1}$ Key Laboratory of Aging and Cancer Biology of Zhejiang Province, Institute of Aging Research, School of Medicine, Hangzhou Normal University, Hangzhou 311121, China; ${ }^{2}$ Department of Public Health and Preventive Medicine, Changzhi Medical College, Changzhi, Shanxi 046000, P. R. China; ${ }^{3}$ Key Laboratory of Regenerative Medicine of Ministry of Education, Guangzhou Regenerative Medicine and Health Guangdong Laboratory, Institute of Aging and Regenerative Medicine, Jinan University, Guangzhou 510632, China;

${ }^{4}$ CAS Key Laboratory of Regenerative Biology and Guangdong Provincial Key Laboratory of Stem Cell and Regenerative Medicine, Guangzhou Institutes of Biomedicine and Health, Chinese Academy of Sciences, Guangzhou, China; ${ }^{5}$ Department of Molecular Biology,

Cell Biology and Biochemistry, Brown University, Providence, RI 02903, USA and ${ }^{6}$ Department of Colorectal Surgery, Sir Run Run Shaw Hospital, Zhejiang University, Hangzhou, China

These authors contributed equally: Ying Wang, Jin-ping Zheng Correspondence: Ying Wang (flashingdancer@163.com) or Zhangfa Song (songzhangfa@zju.edu.cn) or Hu Wang (wanghu19860315@163.com) or Zhenyu Ju (zhenyuju@163.com)

\section{REFERENCES}

1. Ju, Z. et al. Telomere dysfunction induces environmental alterations limiting hematopoietic stem cell function and engraftment. Nat. Med. 13, 742-747 (2007).

2. De Cecco, M. et al. Genomes of replicatively senescent cells undergo global epigenetic changes leading to gene silencing and activation of transposable elements. Aging Cell. 12, 247-256 (2013). 
3. Murnane, J. P. \& Sabatier, L. Chromosome rearrangements resulting from telomere dysfunction and their role in cancer. Bioessays 26, 1164-1174 (2004).

4. Niyongere, S. et al. Heterogeneous expression of cytokines accounts for clinical diversity and refines prognostication in CMML. Leukemia 33, 205-216 (2019).

cc) (i) Open Access This article is licensed under a Creative Commons cc) Attribution 4.0 International License, which permits use, sharing, adaptation, distribution and reproduction in any medium or format, as long as you give appropriate credit to the original author(s) and the source, provide a link to the Creative
Commons license, and indicate if changes were made. The images or other third party material in this article are included in the article's Creative Commons license, unless indicated otherwise in a credit line to the material. If material is not included in the article's Creative Commons license and your intended use is not permitted by statutory regulation or exceeds the permitted use, you will need to obtain permission directly from the copyright holder. To view a copy of this license, visit http://creativecommons. org/licenses/by/4.0/.

(c) The Author(s) 2020 